ISSN 0103-5150

Fisioter. Mov., Curitiba, v. 24, n. 4, p. 665-672, out./dez. 2011 Licenciado sob uma Licença Creative Commons

\title{
Avaliação da mobilização neural sobre o ganho de amplitude de movimento
}

\author{
Assessment of neural mobilization over \\ the gain in range of motion
}

\section{Danilo de Almeida Vasconcelos ${ }^{[a]}$, Lívia Cristina Rodrigues Ferreira Lins ${ }^{[b]}$, Estélio Henrique Martin Dantas ${ }^{[c]}$}

[a] Mestre em Engenharia Biomédica, docente da Universidade Estadual da Paraíba (UEPB), Campina Grande, PB - Brasil, e-mail: davasconcelos@yahoo.com.br

[b] Graduada em Fisioterapia pela Fisioterapia da Universidade Estadual da Paraíba (UEPB), Campina Grande, PB - Brasil, e-mail: livialins9@gmail.com

[c] Doutor em Treinamento Desportivo, Laboratório de Biociências da Motricidade Humana (LABIMH) da Universidade Federal do Estado do Rio de Janeiro (UNIRIO), Rio de Janeiro, RJ - Brasil, e-mail: estelio@cobrase.org.br

\section{Resumo}

Introdução: A mobilização neural (MN) visa a restaurar o movimento e a elasticidade do SN, e é utilizada como método de diagnóstico e tratamento das disfunções de origem neural. Objetivo: Verificar os efeitos imediatos da MN sobre o ganho de amplitude de movimento de extensão do cotovelo (ADMEC) em indivíduos com tensão neural adversa do nervo mediano (TNAm). Metodologia: A amostra foi composta por estudantes universitários, de ambos os sexos, na faixa etária de 17 a 30 anos. Cada indivíduo foi avaliado bilateralmente por meio do teste de tensão do nervo mediano (ULTT1). Nos casos de teste positivo, foi aplicada a MN do nervo mediano e realizada a avaliação goniométrica imediatamente pré e pós-MN. Os dados foram analisados de forma descritiva e inferencial por meio do Teste de Normalidade Kolmogorov-Smirnov, teste $\mathrm{t}$ de Student e teste de Wilcoxon, sendo considerado um nível de significância de 5\%. Resultados: A amostra foi composta por 60 voluntários, com idade média de 21,25 \pm 0,29 anos, estatura média de 1,66 \pm 0,11 metros e peso médio de 63,27 $\pm 1,53 \mathrm{~kg}$. Observou-se ganho estatisticamente significante $(\mathrm{p}<0,001)$ da ADMEC em ambos os membros superiores após a MN, com valores de 31,57 $\pm 20,27^{\circ}$ e 20,53 $\pm 15,27^{\circ}$, pré e pós, respectivamente, no membro superior direito, e $28,68 \pm 22,43^{\circ}$ e $16,57 \pm 15,11^{\circ}$, pré e pós, no 
membro superior esquerdo. Conclusão: Neste estudo, a MN foi capaz de melhorar, com resultados imediatos, a ADMEC de forma significativa.

Palavras-chave: Amplitude de Movimento Articular. Nervo Mediano. Mobilização neural.

\section{Abstract}

Introduction: The neural mobilization (NM) aims to restore movement and elasticity of the NS, and it is used as a method of diagnosis and treatment of disorders of neural origin. Objective: To investigate the immediate effects of NM over the gain in range of motion of elbow extension (ROMEE) in individuals with adverse neural tension (ANT) of the median nerve. Methodology: The sample composed of college students, of both sexes, aged 17 to 30 years. Each subject was assessed bilaterally using the tension test of the median nerve (ULTT1). In cases of positive result of tests the NM was applied to the median nerve and goniometric evaluation performed immediately before and after NM. The data were analyzed using descriptive and inferential Normality Test $f$ Kolmogorov Smirnov, $t$ Student test and Wilcoxon test, considering a significance level of 5\%. Results: The sample consisted of 60 volunteers with a mean age of $21.25 \pm 0.29$ years, mean height of $1.66 \pm 0.11$ meters and mean weighing of $63.27 \pm 1.53 \mathrm{~kg}$. Was observed statistically significant improvement $(p<0.001)$ of ROMEE in both upper limbs after the NM, with values of $31.57 \pm 20.27^{\circ}$ and $20.53 \pm 15.27^{\circ}$, pre and post, respectively, on the right and $28.68 \pm 22.43^{\circ}$ and $16.57 \pm 15.11^{\circ}$, pre and post in the left upper limb. Conclusion: In this study, NM was able to improve, with immediate results, the ROMEE significantly.

Keywords: Range of Motion. Median Nerve. Neural mobilization.

\section{Introdução}

A principal função do Sistema Nervoso (SN) é a condução de impulsos; porém, esta é extremamente dependente da parte mecânica desse sistema e viceversa. A interligação da função mecânica e fisiológica do SN foi reunida no termo neurodinâmica. Se esse sistema está apresentando normalidade neurodinâmica, significa que suas propriedades mecânicas e fisiológicas estão normais $(1,2)$.

Não cabe ao SN somente conduzir impulsos por meio de grandes amplitudes e complexidades de movimento, mas também adaptar-se mecanicamente a esses movimentos, retraindo-se e alongando-se, podendo até mesmo limitar essas amplitudes em certas combinações de movimentos $(1,3)$.

Quando a neurodinâmica está alterada, ocorre o que se denomina de Tensão Neural Adversa (TNA), que consiste numa resposta mecânica e fisiológica anormal quando a amplitude normal do SN e sua capacidade de alongamento são testadas (1).

A atividade adequada do SN depende de sua integridade. 0 comprometimento da mecânica e da fisiologia do SN pode resultar em outras disfunções próprias do mesmo, bem como das estruturas musculoesqueléticas que recebem sua inervação $(1,4)$.
Os nervos periféricos são frequentemente traumatizados, resultando em lesões que trazem como consequência perda ou redução da sensibilidade e da motricidade no território inervado pelos mesmos (5). Entre os diversos nervos periféricos do corpo, o nervo mediano apresenta traumatismos frequentes, resultando em lesões que trazem como consequência a redução da capacidade funcional do individuo (6).

Os exames que avaliam o envolvimento do tecido neural e a presença de TNA vêm sendo alvo de pesquisas. Os testes de restrição de mobilidade do SN são considerados importantes há muitos anos, incluindose nos procedimentos que são empregados em geral para avaliação de distúrbios musculoesqueléticos. Os testes neurodinâmicos envolvem aplicação de um conjunto de movimentos passivos destinados a testar a capacidade de adaptação do tecido neural a diferentes posições funcionais $(7,8)$.

A Mobilização Neural (MN) é uma técnica que tem como objetivo restaurar o movimento e a elasticidade do $\mathrm{SN}$, o que promove o retorno às suas funções normais e a redução do quadro sintomático (9). Embora a MN não seja amplamente conhecida, essa ideia de aplicar um tratamento mecânico para o tecido neural não é nova. Princípios e métodos do alongamento neural já existiam desde o ano 1800 e progressivamente 
foram se aperfeiçoando tanto na teoria quanto na aplicação clínica (10).

Considerando que a TNA é um dos fatores que limita o movimento, presume-se que sua resolução ou diminuição por meio da MN resultaria numa melhor dinâmica neural e, consequentemente, permitiria uma maior ADM. Diante desse contexto, o objetivo desse estudo foi avaliar os efeitos imediatos da mobilização neural do nervo mediano sobre o ganho de amplitude de movimento de extensão do cotovelo (ADMEC) em indivíduos neurologicamente assintomáticos.

\section{Materiais e métodos}

O presente estudo foi do tipo quantitativo de caráter exploratório transversal, realizado no departamento de Fisioterapia da Universidade Estadual da Paraíba (UEPB) no período de outubro de 2009 a julho de 2010. 0 presente trabalho atendeu às normas para a realização de pesquisa em seres humanos e cumpriu as diretrizes da Resolução n. 196/96 do Conselho Nacional de Saúde/MS, sendo o trabalho aprovado pelo Comitê de Ética da Universidade Estadual da Paraíba, n. 0606.0.133.000-09.

A amostra foi do tipo não probabilistica intencional composta por indivíduos de ambos os sexos na faixa etária de 17 a 30 anos. Foram incluídos os indivíduos estudantes do curso de fisioterapia da UEPB devidamente matriculados, neurologicamente assintomáticos, que aceitaram participar da pesquisa voluntariamente e assinaram o termo de consentimento livre e esclarecido. Foram adotados como critérios de exclusão: presença de patologias neurológicas, musculoesqueléticas, reumáticas e tráumato-ortopédicas que pudessem interferir nos resultados do trabalho proposto e/ou qualquer fator de contraindicação para a realização da MN.

Como instrumentos para coleta dos dados, foram utilizados: uma ficha de identificação dos participantes elaborada pelos avaliadores, Inventário de Dominância Lateral de Edimburgo, Estadiômetro (CARCI), balança portátil, goniômetro clínico (CARCI) e uma maca portátil de quiropraxia (MEXQUIRO).

A coleta dos dados foi realizada por dois avaliadores e foi dividida em três momentos: avaliação inicial (pré-mobilização), aplicação da mobilização neural e reavaliação (pós-mobilização neural).

Durante a avaliação inicial, um avaliador (avaliador 1) preencheu a ficha de identificação de cada participante, com dados referentes ao nome, idade, sexo, estatura e peso, e cada indivíduo respondeu ao Inventário de Dominância Lateral de Edimburgo para que o seu coeficiente de dominância lateral fosse determinado.

Em seguida, um segundo avaliador (avaliador 2) posicionou o participante em decúbito dorsal (DD) sobre uma maca para submetê-lo ao Teste de avaliação neurodinâmica do nervo mediano 1 (ULTT1), um teste passivo que avalia a capacidade mecânica e permite o diagnóstico clínico de tensão neural adversa do nervo mediano (TNAm). 0 ULTT1 envolve a aplicação de movimentos, em sequência, de depressão da cintura escapular, abdução do ombro a $90^{\circ}$, rotação lateral do ombro, supinação do antebraço, extensão do punho e dedos, extensão do cotovelo, além de flexão cervical para o lado oposto como manobra de sensibilização (1).

Nesta pesquisa, o ULTT1 foi considerado positivo quando o indivíduo relatou dor e/ou parestesia no trajeto do nervo mediano e apresentou uma redução da ADMEC verificada como uma resistência tecidual pelo avaliador durante o teste.

Nos casos de presença de TNAm, o Avaliador 2 manteve o membro superior do participante na posição que verificou a angulação correspondente à TNAm da ADMEC, enquanto o Avaliador 1 realizou por meio do goniômetro clínico a mensuração do ângulo de extensão do cotovelo, tendo como pontos de referência: o processo estiloide da ulna, epicôndilo medial do úmero e a linha axilar média a fim de identificar a limitação de ADM em graus, pré-mobilização neural do nervo mediano.

Os participantes que apresentaram TNAm foram submetidos ao protocolo de MN para o nervo mediano proposto por Butler (1). O protocolo foi aplicado pelo Avaliador 2 e consistiu em movimentos oscilatórios de elevação da cintura escapular com extensão de punho e dedos e depressão da cintura escapular com punho neutro. Foram realizadas 20 oscilações por minuto durante dois minutos, com um intervalo de 30 segundos para repouso, no membro com TNAm.

Imediatamente após a aplicação da MN, realizouse uma nova avaliação goniométrica, na mesma sequência da avaliação pré-mobilização, a fim de identificar diferenças, em graus, da ADMEC pré e pós-MN do nervo mediano. Os dados da presença de TNAm e os graus de ADM de extensão do cotovelo pré e pós-mobilização neural foram anotados na ficha de 
identificação do participante. Cada indivíduo foi avaliado bilateralmente, sempre iniciando a avaliação e a MN pelo membro superior direito a fim de padronizar o estudo.

A análise dos dados foi feita de forma descritiva e inferencial usando o pacote estatístico SPSS v. 13.0. A normalidade da amostra foi verificada por meio do teste de Kolmogorov-Sminorv, a fim de definir a utilização de testes paramétricos ou não paramétricos nas análises. Para comparar os valores de ADMEC pré e pós-MN, utilizamos, nos dados paramétricos, o teste t de Student, e nos dados não paramétricos, o teste de Wilcoxon. Para todos os testes utilizados, os dados foram considerados estatisticamente significantes para um nível de significância de $5 \%$ para aceitação da hipótese nula.

\section{Resultados}

A amostra foi composta por 60 indivíduos voluntários, 19 (32\%) do sexo masculino e 41 (68\%) do sexo feminino, com idade média de 21,25 $\pm 0,29$ anos, estatura média de $1,66 \pm 0,11$ metros e peso médio de $63,27 \pm 1,53 \mathrm{~kg}$.

Os dados referentes à presença de TNAm identificaram 9 (15\%) indivíduos com TNAm apenas no membro superior direito, 6 (10\%) apenas no membro superior esquerdo, 39 (65\%) em ambos os membros superiores e 6(10\%) indivíduos não apresentaram TNAm, como ilustrado no Gráfico 1.

A correlação entre o sexo dos sujeitos da amostra e a presença de TNAm está demonstrada na Tabela 1.

Em relação ao Índice de Dominância Lateral de Edimburgo, a análise dos dados classificou 2 (3,3\%) indivíduos como canhotos e 58 (96,7\%) como des- tros. Não foi observada correlação entre a presença de TNAm e o índice de dominância lateral dos sujeitos, uma vez que os sujeitos canhotos e a maioria dos destros apresentaram TNAm em ambos os membros superiores sem prevalência de TNAm no membro superior esquerdo e no membro superior direito, respectivamente, como demonstrado na Tabela 2. Porém, a quantidade limitada dos indivíduos da amostra não permite generalizar os dados, não havendo relevância estatística desse resultado.

A avaliação da amplitude de movimento de extensão do cotovelo (ADMEC) do membro superior direito mostrou normalidade dos dados, de acordo com o teste de Kolmogorov-Sminorv. A média da ADMEC pré-MN do nervo mediano direito foi de

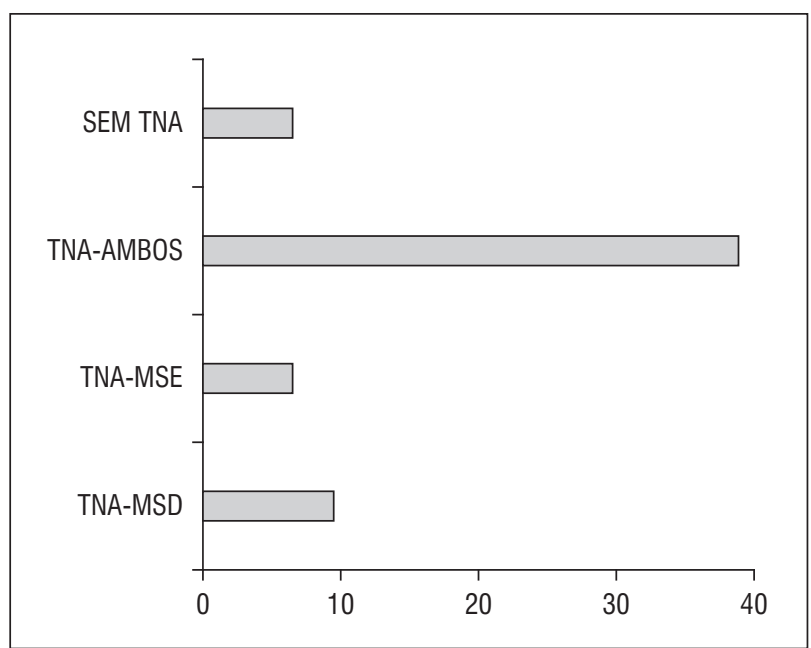

Gráfico 1 - Presença de Tensão Neural Adversa do Nervo Mediano (TNAm)

Legenda: TNA-MSD = TNAm no membro superior direito; TNAMSE = TNAm no membro superior esquerdo; TNAAMBOS = TNAm em ambos os membros superiores; SEM TNA $=$ Membro superior sem TNAm.

Fonte: Dados da pesquisa.

Tabela 1 - Sexo dos sujeitos da amostra em relação à TNAm

\begin{tabular}{llccccc}
\hline & & \multicolumn{5}{c}{ Presença de tensão neural adversa } \\
\cline { 3 - 6 } & & MSE & MSD & MMSS & STNA & Total \\
\hline \multirow{2}{*}{$\begin{array}{l}\text { Sexo dos sujeitos } \\
\text { da amostra }\end{array}$} & Masculino & $2(11 \%)$ & $2(11 \%)$ & $13(67 \%)$ & $2(11 \%)$ & 19 \\
\hline & Feminino & $4(10 \%)$ & $7(17 \%)$ & $26(63 \%)$ & $4(10 \%)$ & 41 \\
\hline
\end{tabular}

Legenda: MSE = Membro superior esquerdo; MSD = Membro superior direito; MMSS = Ambos os membros superiores; STNA = sem tensão neural adversa.

Fonte: Dados da pesquisa. 
Tabela 2 - Relação entre o Índice de dominância lateral e a Presença de TNAm

\begin{tabular}{llccccc}
\hline & & \multicolumn{3}{c}{ Presença de tensão neural adversa } & \multirow{2}{*}{ Total } \\
\cline { 3 - 6 } & & MSE & MSD & MMSS & \multicolumn{1}{c}{ STNA } & 0 \\
\hline \multirow{2}{*}{$\begin{array}{l}\text { Índice de } \\
\text { dominância lateral }\end{array}$} & Canhoto & $0(0 \%)$ & $0(0 \%)$ & $2(100 \%)$ & $0(0 \%)$ & 2 \\
& Destro & $6(10 \%)$ & $9(16 \%)$ & $37(64 \%)$ & $6(10 \%)$ & 58 \\
\hline & Total & $6(10 \%)$ & $9(15 \%)$ & $39(65 \%)$ & $6(10 \%)$ & 60 \\
\hline
\end{tabular}

Legenda: MSE = Membro superior esquerdo; MSD = Membro superior direito; MMSS = Ambos os membros superiores; STNA $=$ sem tensão neural adversa.

Fonte: Dados da pesquisa.

$31,57 \pm 20,27^{\circ}$ e pós-MN de $20,53 \pm 15,27^{\circ}$, demonstrando um aumento da extensão do cotovelo direito de $11,04^{\circ}$ após a aplicação da MN com uma diferença estatisticamente muito significante $(\mathrm{p}<0,01)$ pelo teste $t$ de Student para dados pareados, como demonstrado no Gráfico 2.

Os dados da ADMEC do membro superior esquerdo mostraram-se não paramétricos pelo teste de Kolmogorov-Sminorv. A média foi de 28,68 $\pm 22,43^{\circ}$ e $16,57 \pm 15,11^{\circ}$ pré e pós-MN do nervo mediano esquerdo, respectivamente, com um aumento de $12,11^{\circ}$ da extensão do cotovelo esquerdo após a MN, apresentando diferença estatística muito significante $(\mathrm{p}<$ 0,01 ) pelo teste de Wilcoxon, como demonstrado no Gráfico 3.

\section{Discussão}

O SN pode ser comparado à letra "H", no sentido em que une todas as partes do corpo. É considerado um trato tecidual contínuo, o que significa que uma tensão aplicada em qualquer parte do " $\mathrm{H}$ " pode ser disseminada em duas direções. Desse modo, é possível compreender como o movimento de uma parte do corpo produz um efeito de ampliação ou de restrição em outra $(11,12,13)$.

A capacidade dos músculos de contrair e relaxar com o mínimo de resistência em todas as suas amplitudes de movimento depende, além dos impulsos motores provenientes do sistema nervoso, de três fatores: a elasticidade e a completa extensibilidade dos músculos, amplitude completa das articulações e um sistema nervoso livremente móvel e extensível (14).

Uma lesão nervosa implica alterações das propriedades mecânicas e fisiológicas do SN que, por sua vez, sustentam ou agravam a lesão. Tais lesões

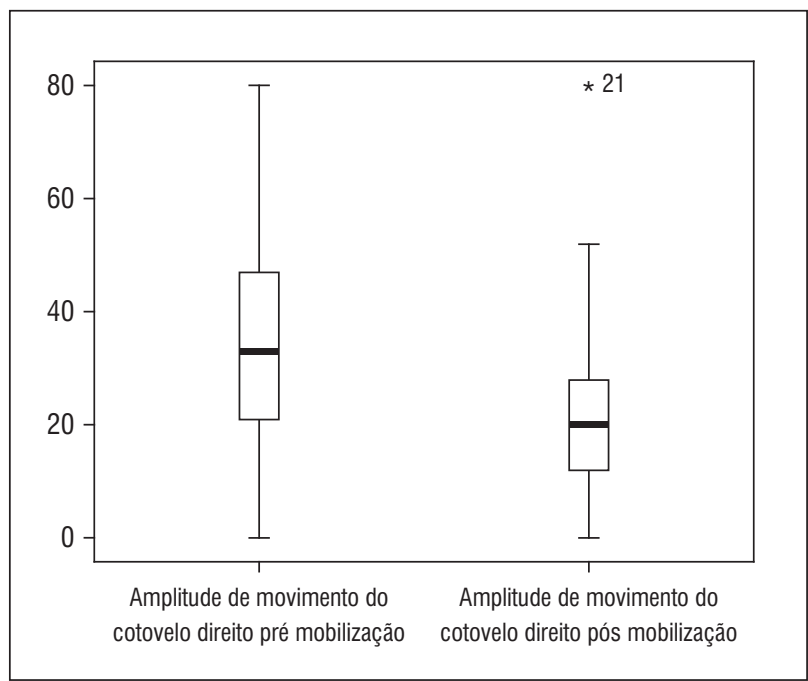

Gráfico 2 - Variação da ADMEC do membro superior direito pré e pós-MN do nervo mediano direito

Fonte: Dados da pesquisa.

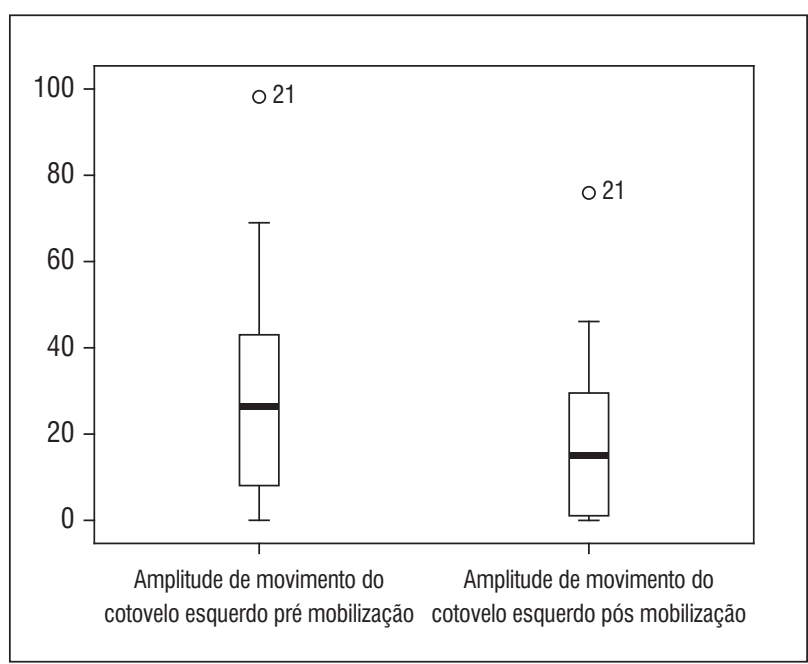

Gráfico 3 - Variação da ADMEC do membro superior esquerdo pré e pós-MN do nervo mediano esquerdo Fonte: Dados da pesquisa. 
podem resultar em disfunções nas estruturas que recebem sua inervação. Como consequência, estruturas músculo-esqueléticas podem estar comprometidas numa disfunção de origem neural (4).

A avaliação do envolvimento neural dos membros superiores é feita por meio de alguns testes, denominados Upper Limb Tension Tests (ULTTs). São destinados a aplicar estresse sobre as estruturas neurológicas do membro superior, embora na verdade estresse seja posto em todos os tecidos do membro superior. 0 tecido neurológico é diferenciado quando se é adicionado um componente sensibilizador, como, por exemplo, pedir ao paciente que flexione o pescoço para o lado contralateral ao lado testado $(12,14)$. No presente estudo, foi abordado o ULTT1, utilizado para avaliar o nervo mediano, comumente acometido por lesões.

Os testes são considerados positivos quando o indivíduo apresenta diminuição da amplitude de movimento (ADM). Sintomas dolorosos e de alongamento profundo são reproduzidos quando a resposta no lado envolvido varia unilateralmente entre respostas normais e/ou quando há diferenciação estrutural de uma fonte neurogênica $(16,17)$.

Silva e Salgado (18) realizaram um estudo com um paciente portador de síndrome dolorosa miofascial, associando o ULTT1 e o teste da elevação da perna estendida (SLR), e verificaram que o teste reproduziu os sintomas anteriormente referidos pelo paciente.

O movimento de extensão da articulação do cotovelo é considerado como o retorno da flexão, ou seja, $145^{\circ}-0^{\circ}(20)$. Portanto, quanto mais próximo de $0^{\circ}$ o movimento de extensão, melhor a ADMEC. A redução da amplitude de movimento pode ser encontrada como resposta aos ULTTs $(1,19)$.

Neste estudo, a utilização do ULTT1 demonstrou um déficit na ADMEC em indivíduos neurologicamente assintomáticos, com uma média de $31,5^{\circ} \pm 2,6^{\circ}$ no membro superior direito e de $28,6^{\circ} \pm 2,8^{\circ}$ no membro superior esquerdo, verificado por meio da avaliação goniométrica.

$\mathrm{O}$ tratamento da TNA é feito por meio da $\mathrm{MN}$, a qual tem como objetivo restaurar o movimento e a elasticidade do SN, melhorando a neurodinâmica e restabelecendo o fluxo axoplasmático, promovendo a homeostasia dos tecidos nervosos $(1,12)$.

Apesar de a técnica não atuar diretamente em músculos e fáscias, é observado ganho de amplitude de movimento após sua utilização (9). No presente estudo foram verificados os efeitos imediatos da
MN do nervo mediano sobre o ganho de amplitude de movimento de extensão do cotovelo em indivíduos neurologicamente assintomáticos. Obteve-se ganho médio de $11,04^{\circ}$ no membro superior direito, variando de uma média de $31,57^{\circ}$ pré-mobilização para $20,53^{\circ}$ pós-mobilização, e $12,11^{\circ}$ no membro superior esquerdo, variando de $28,68^{\circ}$ para $16,57^{\circ}$ pré e pós-mobilização, respectivamente, verificandose que essa técnica foi eficaz para aumentar a amplitude de movimento nos casos de TNA.

A MN tem sido utilizada no tratamento das mais diversas patologias do sistema nervoso, bem como das disfunções dos tecidos por ele inervados (9).

Um estudo feito por Santos (21) demonstrou redução da dor e melhora da coordenação durante a escrita, após a mobilização no nervo mediano, em um paciente portador da síndrome do escrivão. Guelfi (22) utilizou a técnica de mobilização do sistema nervoso, por quatro semanas, na posição de SLR, em um paciente portador de siringomielia e observou redução das dores sentidas nos pés e no membro superior direito, avaliadas utilizando-se da escala de dor.

Estudos evidenciaram ganho significativo de amplitude de movimento após a aplicação da MN (23, $24,25)$. Estudos que aplicaram a MN em pacientes, com sintomas de cervicobraquialgia, verificaram ao final do tratamento um aumento da amplitude de movimento da coluna cervical $(26,27)$.

Vasconcelos (28) aplicou a técnica de MN em pacientes com lombociatalgia e observou melhora gradual das amplitudes de movimento. Machado e Bigolin (29) verificaram um incremento da flexibilidade da cadeia muscular posterior em pacientes com lombalgia crônica após a mobilização neural. Já Boeing (30) verificou melhora estatisticamente significativa na redução do quadro álgico em pacientes com lombociatalgia; entretanto, a mobilidade lombar e a sensibilidade não apresentaram melhora significativa.

\section{Considerações finais}

Pesquisas têm sido desenvolvidas com objetivos de avaliar a eficácia da MN como método de diagnóstico e de tratamento nas mais variadas disfunções que afetam esse sistema e as estruturas por ele inervadas.

Neste estudo, a mobilização neural do nervo mediano demonstrou-se como uma técnica eficaz para o ganho de amplitude de movimento de extensão 
do cotovelo em indivíduos assintomáticos. Os resultados mostraram-se satisfatórios, porém só foram avaliados os efeitos imediatos da técnica, sugerindo-se o desenvolvimento de estudos que avaliem os efeitos a longo prazo da mobilização neural.

A literatura ainda é escassa no que se refere a esse tema, e especialmente quanto à aplicação da MN nos membros superiores e sua influência sobre a ADM. Sendo assim, se faz importante a realização de mais estudos com objetivos semelhantes a fim de comprovar cientificamente os benefícios dessa técnica. Dessa forma, promover a divulgação dos efeitos da MN no âmbito da fisioterapia.

\section{Referências}

1. Butler DS. Mobilização do sistema nervoso. São Paulo: Manole; 2003.

2. Cerqueira MP, Reis AMO. Mobilização neural no tratamento da síndrome do túnel do carpo. Ter Man. 2003; 2(2):82-5.

3. Stokes M. Neurologia pra fisioterapeutas. São Paulo: Premier; 2000.

4. Marizenck S. Mobilização neural: aspectos gerais. [acesso 7 jul. 2010]. Disponível em: http://www. terapiamanual.com.br/site/noticias/arquivos/2009 12101725220.artigo_7.pdf.

5. Machado A. Neuroanatomia funcional. São Paulo: Atheneu; 2002.

6. Kisner C, Colby AL. Exercícios terapêuticos: fundamentos e técnicas. São Paulo: Manole; 2005.

7. Davies PM. Hemiplegia: tratamento para pacientes após AVC e outras lesões cerebrais. Barueri: Manole; 2008.

8. Salgado ASI. Escola de terapia manual e postural: neuromeningea, craniomandibular e otorrinolaringologia. Londrina: Leal; 2004.

9. Oliveira HFO Junior, Teixeira AH. Mobilização do sistema nervoso: avaliação e tratamento. Fisioter Mov. 2007;20(3):41-53.

10. Kostopoulos D. Treatment of carpal tunnel syndrome: a review of the non-surgical approaches with emphasis in neural mobilization. Journal of Bodywork and Movement Therapies. 2004;8:2-8.
11. Jesus CS. A mobilização do sistema nervoso e seus efeitos no alongamento da musculatura ísquiotibial. Ter Man. 2004;2(4):162-5.

12. Butler DS. Adverse mechanical tension in the nervous system: a model for assessment and treatment. Aust J Physiother. 1989;35(4):227-38.

13. Beleski RC. Verificação da presença de tensão neural nas cervicobraquialgias por meio dos testes de tensão neural para nervo mediano e radial. Ter Man. 2004; 2(4)182-5.

14. Shacklock MO. Positive upper limb tension test in a case of surgically proven neuropathy: analysis and validity. Man Ther. 1996;1(3):154-61.

15. Zamberlan AL, Kerppers II. Mobilização neural como um recurso fisioterapêutico na reabilitação de pacientes com acidente vascular encefálico: revisão. Rev Salus. 2007;1(2):185-91.

16. Magee DJ. Avaliação musculoesquelética. Barueri: Manole; 2005.

17. Mahmud MAI, Merlo ARC, Gomes I, Becker J, Nora DN. Relação entre tensão neural adversa e estudos de condução nervosa em pacientes com sintomas da síndrome do túnel do carpo. Arq Neuropsiquiatr. 2006;64(2-A):277-82.

18. Silva RBX, Salgado ASI. Fisioterapia manual na síndrome dolorosa miofascial. Ter Man. 2003;2(2):74-77.

19. Martins DF. Respostas normais do teste neurodinâmico para membro superior com tendência ao nervo mediano 2a (TNMS2a) [trabalho de conclusão de curso]. Tubarão: Universidade do Sul de Santa Catarina; 2005.

20. Marques AP. Manual de goniometria. Barueri: Manole; 2003.

21. Santos VR. A influência da mobilização do sistema nervoso na cãimbra do escrivão. Ter Man. 2004;2(4): 166-71.

22. Guelfi MD. A influência da mobilização do sistema nervoso em um indivíduo portador de siringomielia. Ter Man. 2004;2(4):158-61.

23. Marcolino AM, Barbosa RI, Fonseca MCR, Mazzer N, Elui VMC. Reabilitação fisioterapêutica na lesão do plexo braquial: relato de caso. Fisioter Mov. 2008; 21(2):53-60. 
24. Smaniotto ICG, Fonteque MA. A influência da mobilização do sistema nervoso na amplitude de movimento da flexão do quadril. Ter Man. 2004;2(4):154-7.

25. Abreu ACD, Godinho IO, Aquino MS. Efeito da técnica neurodinâmica na mobilidade da coluna lombar. Ter Man. 2007;5(22):322-5.

26. Brismé JM, Phelps V, Sizer P. Differential diagnosis and treatment of chronic neck and upper trapezius pain and upper extremity paresthesia: a case study involving the management of an elevated first rib and uncovertebral joint dysfuntion. The Journal of Manual and Manipulative Therapy. 2005;13(2):79-90.

27. Ribeiro CD, Braga IO, Silva JR Junior, Macedo LC, Silva WR Junior, Vasconcelos DA. Mobilização neurodinâmica no tratamento fisioterapeutico da cervicobraquialgia. In: Vasconcelos DA, Silva, JR Junior, Silva MSB. (Org.). Fisioterapia baseada em evidências: fisiociência. Campina Grande: EDUEP; 2008. p. 237-59.
28. Vasconcelos B. A eficácia da mobilização neural no tratamento do quadro álgico em pacientes com lombociatalgia [trabalho de conclusão de curso]. Cascavel: Faculdade Assis Gurgacz; 2007.

29. Machado GF, Bigolin SE. Estudo comparativo de casos entre a mobilização neural e um programa de alongamento muscular em lombálgicos crônicos. Fisioter Mov. 2010;23(4):545-54.

30. Boeing M. Análise da eficácia de técnicas de mobilização neural para pacientes com lombociatalgia [trabalho de conclusão de curso]. Cascavel: Universidade Estadual do Oeste do Paraná; 2004.

Recebido: 09/11/2010

Received: 11/09/2010

Aprovado: 30/05/2011

Approved: 05/30/2011 